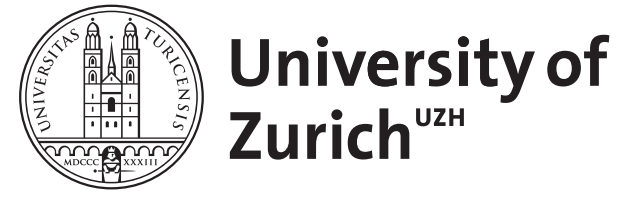

\title{
Publish your case report
}

Schneemann, Markus ; Ruggieri, Fabio

DOI: https://doi.org/10.1024/1661-8157/a001229

Posted at the Zurich Open Repository and Archive, University of Zurich ZORA URL: https://doi.org/10.5167/uzh-84421

Journal Article

Accepted Version

Originally published at:

Schneemann, Markus; Ruggieri, Fabio (2013). Publish your case report. Praxis, 102(5):253-259; quiz 60. DOI: https://doi.org/10.1024/1661-8157/a001229 


\section{Publish Your Case Report!}

"Wat den Einen sin Uuhl is, is den Annern sin Nachtigal"

Fritz Reuter, Ut mine Stromtid

Der klinische Fallbericht (im Folgenden: Case Report) hat eine lange Tradition, die sich bis in die Antike zurückverfolgen lässt. Neben der Biographie berühmter Heiler und Ärzte ist er das älteste schriftliche Zeugnis mit medizinischem Inhalt. In jüngerer Zeit ist er allerdings etwas in Misskredit geraten. Im Zeitalter industrie-gesponserter, randomisierter Studien erscheint die Beschreibung einer einzelnen Patientengeschichte zu billig und zu banal. Auch generiert ein Einzelfall in der Regel wenige Zitate, und wird deshalb von vielen Zeitschriften abgelehnt, da Case Reports den Impact factor negativ beeinflussen. Ein prominentes Beispiel ist das Swiss Medical Weekly, welches in ihren Instructions for Authors explizit schreibt: „The submission of case reports is discouraged". Nichtsdestotrotz ergibt eine Suche im PubMed knapp über eine halbe Million Resultate beim Suchwort „Case Report“ für die letzten zehn Jahre. Seit 2007 sind neue Zeitschriften entstanden, die sich exklusiv dem Genre des Case reports widmen (Abb. 2). Um den Zugang möglichst global und günstig zu machen, sind die meisten dieser neuen Publikationsorgane „open access“ und nur noch digital, also „paperless“ über das Internet zugänglich. Die Sprache ist bei den meisten Englisch, deshalb haben wir auch bewusst unseren Beitrag mit einem englischsprachigen Titel versehen: „Publish your Case Report!“ (1). Unser Artikel möchte den Leser davon überzeugen, dass Case Reports einen wissenschaftlichen und didaktischen Wert, vor allem für die Autoren selber, haben, wie auch Johann Steurer jüngst in dieser Zeitschrift ausgeführt hat (2). In die gleiche Richtung zielt ein Aufruf von Richard Smith, dem ehemaligen Herausgeber des British Medical Journal, der 2009 einen Aufruf an alle Hausärzte betitelte: „Why GPs should write case reports" (3). 
Abb. 1:

Neue Krankheiten, ungewöhnliche Präsentationen

Ideen, Hypothesen zur Pathogenese von Krankheiten

Neue unerwünschte Arzneimittelwirkungen

Langzeitverläufe

Multimorbidität

Diagnostische und therapeutische Dilemmata

Medizinische Fehler und Beinahe-Fehler

Gute Geschichten (narrative medicine)

Legende: Ausgewählte mögliche Themen zur Veröffentlichung als Case Report 
Abb. 2:

In English:

Journal of Medical Case Reports (JMCR)

BMJ Case Reports

BMC Research Notes

Case Reports in Medicine

Grand Rounds

Open Access Case Reports

Wiener Klinische Wochenschrift

In Deutsch:

PRAXIS - Schweizerische Rundschau für Medizin

Schweizerisches Medizin-Forum

Deutsche Medizinische Wochenschrift

Wiener Klinische Wochenschrift

In Französisch:

PRAXIS - Revue suisse de la médecine

Forum Médicale Suisse

Revue Médicale Suisse

La Revue de Médecine Interne

In Italienisch:

Recenti Progressi in Medicina

La Clinica Terapeutica

Legende: Ausgewählte Zeitschriften zur Veröffentlichung von Case Reports /

Fallberichten aus der Allgemeinen Innere Medizin 
Abb. 3:

Welcher Fall

What is the message?

Welche Form (Bericht, Letter, Coup d'oeil, Bild)

Welches Journal

Einverständniserklärung

Koautoren

Finanzielles

Do a test run: Posterpräsentation (zum Beispiel: SGIM Jahresversammlung)

Schreibstil: KISS - Prinzip!

Abgelehnt? Nicht Entmutigen Lassen!

Legende: Checkliste wichtiger Punkte für die Publikation eines Case Reports 
Abb. 4:

Action Research: Reflexion und Metakognition

Empathie mit dem individuellen Patienten

Lebenslanges Lernen = Become a better doctor!

Teilnahme am wissenschaftlichen Diskurs

Narrative competence

Legende: Persönlicher Nutzen beim Schreiben von Case Reports 
Im Artikel verwendete Abkürzungen

BMC, Bio Med Central; BMJ, British Medical Journal; JMCR, Journal of Medical Case

Reports; SGIM, Schweizerische Gesellschaft für Innere Medizin 


\section{1) Mögliche Themen für Case Reports}

Der praktizierende Arzt im Spital und in der Praxis wird regelmässig mit neuen Patienten, neuartigen Beschwerden, unklaren Symptomen und neuen Medikamenten konfrontiert. Dies macht auch zum grossen Teil die Faszination und Freude an unserem Beruf aus, dass wir ständig und stetig mit Neuem und Unbekanntem zu tun haben. Gerade der Hausarzt als primäre Anlaufstelle sieht häufig neue Erkrankung als Erster, und ist deshalb prädestiniert für die Beschreibung oder zumindest die Meldung neuer Erkrankungen. Denken Sie nur an den Beginn der HIV-Epidemie mit den unklaren Pneumocystis-Pneumonien Anfang der 80er Jahre oder an SARS oder die Grippeepidemien der letzten Jahre, um nur die spektakulärsten Erkrankungen aus dem Bereich der Infektionen der letzten 30 Jahre zu erinnern. In Abbildung 1 haben wir ein paar wichtige Themenfelder aufgelistet, die für Case Reports geeignet sind.

a) Neue Krankheiten und ungewöhnliche Präsentationen

"always note and record the unusual. . .Publish it. Place it on permanent record as a short, concise note. Such communications are always of value"

\section{William Osler}

Die wohl wichtigste Funktion des Case Reports ist die Erstbeschreibung einer Erkrankung. Dies führte in der Vergangenheit häufig auch zur Verewigung des Erstbeschreibers, indem der Name als Eponym verwendet wird, wie zum Beispiel beim Morbus Alzheimer, Morbus Behçet oder Morbus Whipple. Bei der Entdeckung einer neuen Epidemie, wie in jüngerer Zeit der Vogelgrippe, Schweinegrippe oder EHEC, sind Einzelfallberichte essentiell, und geradezu eine Verpflichtung für jeden Arzt, dies zu publizieren.

Zu den neuen Krankheiten gehören auch in erweitertem Sinne ungewöhnliche

Präsentationen und Manifestationen bereits bekannter Erkrankungen, wie zum Beispiel eine Miktionssynkope bei einem Phäochromozytom der Prostata. Aus kritischen Blickwinkel sind diese Beschreibungen Skurrilitäten im Sinne Johann Steurers, bereichern aber unser Bild von der Vielfalt der Medizin und der Natur: Jeder neuentdeckte Käfer ist eine Bereicherung und einer Beschreibung wert, auch wenn er vom Aussterben bedroht ist oder bereits ausgestorben ist.

b) Ideen, Hypothesen zur Pathogenese von Krankheiten

Überlegungen, wie Krankheiten entstehen bzw. verlaufen, anhand eines Einzelfalles sind häufig sehr interessant und generieren Hypothesen für weiterführende Studien. Dies wäre dann ganz im Sinne der Popperschen Theorie, wie Forschung funktionieren soll bzw. funktioniert, nämlich eine Hypothese wird formuliert und durch Experimente bzw. Studien falsifiziert. Es gibt auch ein eigenes Journal, Medical Hypotheses, welches sich ausschliesslich diesem Aspekt widmet.

\section{c) Neue unerwünschte Arzneimittelwirkungen}

Eine weitere unverzichtbare Funktion des Case Reports ist die Veröffentlichung von Nebenwirkungen medizinischer Behandlungen und Interventionen. Im Zusammenhang mit Medikamenten veröffentlichten Naranjo et al. eine „Adverse Drug Reaction Probability Scale“, die, wenn auch nicht unumstritten, bis heute Verwendung findet bei der Beurteilung von unerwünschten Arzneimittelwirkungen in Postmarketing Analysen (http://farmacologiaclinica.info/scales/Naranjo scale). Für das Auftreten von Lebertoxizität 
gibt es in den U.S.A. das DILIN = drug induced liver injury network

(https://dilin.dcri.duke.edu), welches retrospektiv und prospektiv Daten sammelt. Ein wichtiges prominentes Beispiel in der Vergangenheit war das Auftreten neuartiger Missbildungen nach der Einnahme von Thalidomid während der Schwangerschaft. Ein aktuelles prominentes Beispiel ist das Auftreten von Lebertoxizität durch ein modernes Antikoagulans, Ximelagatran, welches schlussendlich 2006 zum Rückzug des Produktes geführt hat. Ein anderes Beispiel für eine seltene, unerwartete Toxizität finden Sie im konkreten Fallbeispiel zu diesem Artikel.

\section{d) Langzeitverläufe}

Die Beschreibung von Einzelfällen im Langzeitverlauf über fünf, zehn oder noch mehr Jahre stellen ein unserer Meinung nach noch viel zu wenig genütztes Potential des Case Reports dar, und wäre eine Domäne der Hausarztmedizin. Wie spannend und lehrreich ist bzw. wäre die Geschichte eines Diabetikers, eines Hypertonikers über die letzten 30 Jahre! Mit welchen Medikamenten wurden Sie behandelt, wie waren die Verläufe. Die fehlenden Daten über längere Zeit sind eine grosse Schwäche der modernen randomisierten Studien.

e) Multimorbidität

Eine weitere grosse Schwäche der modernen Studien sind die Ausschlusskriterien. Viele unserer Patienten, insbesondere jenseits der sechsten Lebensdekade werden ausgeschlossen wegen zu vieler Komorbiditäten, die als lästige Kovariablen bewertet werden. Im realen Leben eines Hausarztes oder Allgemeininternisten sind multimorbide Patienten mit mehr als zwei aktiven chronischen Erkrankungen aber der Regelfall. Auch hier sehen wir eine noch wenig genützte Chance für Case Reports. Michael Kidd, der Herausgeber des JMCR und der neuen Cases Database (http://www.casesdatabase.com) unterstreicht in diesem Zusammenhang die Wichtigkeit von Case Reports: Wir bräuchten nicht nur „Evidence - based practice“, sondern vielmehr auch „Practice - based evidence“.

\section{f) Dilemmata}

Heutzutage sollen Guidelines und auf Studien basierende Evidenz unsere Diagnostik und Therapie leiten. Wie bei der Multimorbidität erwähnt, basieren diese Empfehlungen auf Monomorbiditäten und Studien, bei denen unsere realen, oft multimorbiden Patienten nicht vorkommen. Nun bringen verschiedene Krankheiten jeweils verschiedene Guidelines hervor, die miteinander in Konkurrenz stehen. Nehmen Sie zum Beispiel den typischen Fall eines Patienten mit einem arteriellen stent und chronischem Vorhofflimmern, der blutet. Was machen Sie primär an Antiaggregation und Antikoagulation und welches Medikament heben Sie wie auf bei einer Blutung?

\section{g) Medizinische Fehler und Beinahefehler}

Im Zeitalter von obligatorischen CIRS (critical incident reporting systems) in den modernen Spitälern und dem epochemachenden Bericht des Institute of Medicine von 2000, „To Err Is Human", sind Fehler im medizinischen Altag weniger tabu. Jedoch sind einzelne Geschichten über solche Ereignisse in der Fachliteratur rar, weil kein Arzt gerne über Fehler sprechen möchte. Ein gutes Beispiel hierfür sind die Meldungen der Komplikationenliste der Schweiz von Max Stäubli. Publiziert wurden hieraus zum Beispiel mehrere, zum Teil tödliche Blutungskomplikationen nach der Applikation von subkutanem Heparin in die Bauchwand. In diesem Kontext mögen wir uns daran erinnern, dass jede Wissenschaft vor allem durch Fehler und Irrtümer und dem Lernen daraus sich entwickelt, wie dies Anfang des 20. Jahrhunderts bereits Marcel Proust formulierte: 
„De sorte que croire à la médecine serait la suprême folie, si n'y pas croire n'en était pas une plus grande, car de cet amoncellement d'erreurs se sont dégagées à la longue quelques vérités."

Marcel Proust

h) Gute Geschichten (Narrative Medicine)

Das Erzählen von Geschichten in der Medizin erlebt durch Kollegen wie Rita Charon und Allan Peterkin ein Revival, vermutlich als eine Kompensation unseres zunehmend technisierten und anonymisierten Umganges mit Patienten und ihren Krankheiten. Die sogenannte „narrative medicine“ möchte u.a. dem Individuum, dem Menschen hinter den „Fällen“ wieder ein humanes Gesicht geben. Auch wir als Ärzte sollen uns selbst als Menschen äussern und wahrgenommen werden.

„Narrative studies, many physicians are beginning to believe, can provide the "basic science" of a story-based medicine that can honor the patients who endure illness and nourish the physicians who care for them."

Rita Charon

Die hier kurz skizzierten für Case Reports geeigneten Rubriken brauchen ein jeweils geeignetes Forum zur Publikation. Eine Auswahl geben wir in Abbildung 2. Bei Schwierigkeiten oder Unklarheiten sind wir gerne bereit Sie zu beraten (siehe Korrespondenzadressen).

\section{2) Praktischer Leitfaden zur Publikation eines Case Reports}

In Abbildung 3 möchten wir dem Leser einen Leitfaden an die Hand geben: Wie gehe ich vor, wenn ich einen Case Report veröffentlichen will. Im Fall werden wir dann ein konkretes Beispiel aus unserem Alltag skizzieren.

a) Welcher Fall? What is the message of your Case Report?

Notieren Sie sich spannende Fälle, bemerkenswerte Vorfälle in ein handliches Notizbuch, welches Sie immer bei sich tragen. Wie wir im Spital oder in der Sprechstunde haben Sie ein DINA6 grosses gelbes Büchlein in der Kitteltasche bereit. Dort tragen Sie Datum, Name und beobachtetes Ereignis hinein für Ihren Case Report. Sehr hilfreich ist es schon hier, wenn Sie sich in nur einem Satz aufschreiben, was sie mitteilen wollen: „What is the message of my Case Report"? In Ihrem Begleitbrief zur Einreichung bei der Zeitschrift, im sogenannten Cover letter müssen Sie beschreiben, was Ihre Message ist, und warum Ihr Case Report veröffentlicht werden muss.

b) Welches Journal und welches Format?

Überlegen Sie sich zuerst, in welcher Sprache Sie schreiben möchten. Am besten benützen Sie das Englische, falls Sie aber lieber in Ihrer Muttersprache publizieren möchten, ist das auch möglich. Die mögliche internationale Leserschaft reduziert sich dann allerdings substantiell. In Abbildung 2 haben ein paar Zeitschriften aufgelistet, die Case Reports veröffentlichen. Je nach Journal gibt es verschiedene Rubriken / Formate, in denen Sie ihren Fallbericht veröffentlichen können. Coup d'oeil und Images sind dabei geeignet für kurze Mitteilungen, spannende Bilder oder Befunde. Wie später noch erwähnt, ist es essentiell, dass sie sich an das vom Journal vorgegebene Format halten. 


\section{c) Einverständniserklärung}

Unverzichtbar ist es heute, dass Sie vom Patienten oder seinen Rechtsvertretern eine Einverständniserklärung besorgen. Dies geschieht am besten in schriftlicher Form und idealerweise in der Muttersprache des Patienten. Viele Journals, wie zum Beispiel auch das JMCR oder das BMJ Case Reports haben vorgedruckte Formulare in diversen Sprachen auf ihrer homepage zum herunterladen und Ausdrucken.

\section{d) Koautoren?}

Ein wichtiges und heikles Thema ist: Wer ist Mitautor? Idealerweise sind Sie als Schreibender und korrespondierender Autor der Erst- (first author) oder der Letztautor (senior author). Koautoren sollten die sog. Vancouver Kriterien („Uniform Requirements“) erfüllen, das heisst, substantiell und inhaltlich zum Case Report beigetragen haben. Der Chef gehört nicht aufs paper, nur weil er ihr Chef ist! Auch andere geschenkte Autorenschaften (gift authorship) widersprechen den international fast überall anerkannten Vancouver Kriterien. Ebenso problematisch sind ghost authors: Das sind Autoren, die nicht genannt werden möchten, obwohl sie beigetragen haben. Dies kann der Fall sein bei Interessenkonflikten, wie bei Angestellten der Industrie. Insgesamt sollte auch die totale Anzahl an Autoren nicht zu gross sein. Die Anfertigung und Befundung eines Röntgenbildes berechtigt zum Beispiel noch nicht zu einer Koautorenschaft. Auf der anderen Seite haben wir die Erfahrung gemacht, dass es besser ist, kulant zu sein, wenn eine(r) unbedingt Koautor sein will. Dafür dürfen sie dann aber auch etwas Substantielles von ihm verlangen. Verlangt ist Fingerspitzengefühl. Viele ihrer guten Kollegen werden sie plötzlich von einer ganz neuen Seite kennenlernen, wenn sie mit Ihnen etwas gemeinsam publizieren wollen.

\section{e) Wer bezahlt?}

Publizieren ist ein Geschäft. Das bedeutet, es kostet in den meisten Fällen Geld, wenn Sie ihren Fall veröffentlichen wollen. Klären sie das mit ihren Koautoren, ihrer Klinik usw. ab, wer was bezahlt. Meist bewegt es sich in der Grössenordnung von zweihundert bis knapp über 1000 Franken, Euro, Dollars oder Pounds je Case Report. Einige Universitätsspitäler sind als Institution bei BMC oder dem BMJ registriert, damit Angestellte zu einem reduzierten Preis publizieren können.

\section{f) Do a test run: Posterpräsentation!}

Als Kaderärzte einer Klinik haben wir mit Posterpräsentationen, zum Beispiel bei den Jahresversammlungen der Fachgesellschaften in der Schweiz, sehr gute Erfahrungen gemacht. Intern nennen wir das einen „Test drive“ für die mitschaffenden Kolleginnen und Kollegen. Sie haben als Autorin ein erstes Erfolgserlebnis, sie sind ein aktiver Teil des Kongresses. Auch als Mentor ist es sehr schön zu sehen, wie sich junge Kolleginnen und Kollegen freuen, wenn Sie vor ihrem Poster stehen. Ausserdem erhalten sie wichtige und gute Feedbacks von Kollegen zu ihrem Fall. Dies wiederum hilft innen dann bei der schriftlichen Formulierung des Case Reports zur schriftlichen Publikation. Sie erhalten zudem einen ersten Eindruck, ob sie oder ihre Koautoren es schlussendlich schaffen werden, den Fallbericht bis zur Publikation zu bringen. Aus unserer Erfahrung sehen die Erfolgschancen grob so aus: Einer von zwei Kollegen schaffen es bis zum Poster, und von diesen jeder vierte bis fünfte zu einem schriftlich veröffentlichten Case Report.

g) Schreibstil: KISS - Prinzip!

Ein häufiges, unter Schriftstellern wohl bekanntes und gefürchtetes Problem ist die Schreibblockade. Viele von innen kennen das sicher noch aus ihrer Schul- oder Studienzeit. Durch die Bologna-Reform in der Schweiz müssen nun alle Medizinstudenten für ihren Masterabschluss in Medizin eine Masterarbeit fertigstellen. Das sollte für die Zukunft 
bedeuten, dass jeder praktizierender Arzt auch ohne medizinischen Doktortitel schon etwas geschrieben oder gar publiziert hat. Zusätzlich kommt erschwerend hinzu, in einer Fremdsprache schreiben zu müssen. Hierfür raten wir innen, schreiben sie in ihrer Muttersprache, bevor sie es gar nicht versuchen wollen. Oder suchen Sie sich Hilfe bei ihren Koautoren, die vielleicht besser sind im Englisch. Ein wichtiges Prinzip beim Schreiben stammt aus den angloamerikanischen Hinweisen für das Schreiben von Case Reports. Halten Sie sich an das vor allem in der Software Industrie häufig zitierte KISS - Prinzip von Kelly Johnson, einem U.S. amerikanischen Flugingenieur: Keep It Simple, Stupid! Das heisst vor allem: Kurze Sätze! Und nur das Wesentliche.

\section{h) Abgelehnt? Nicht Aufgeben!}

Bei allen Schwierigkeiten, auf die Sie treffen könnten, ist die grösste, wie Sie umgehen mit einer Ablehnung. Meistens werden die Artikel von zwei bis drei Experten beurteilt. Dabei handelt es sich in der Regel um Arztkollegen, die sich auf dem Fachgebiet ihres Case Reports auskennen (mehr oder weniger). Diese sogenannten peers werden von den Herausgebern des Journals, an das sie ihren Artikel geschickt haben, ausgewählt. Ein prominenter Kritiker des peer review Prozesses ist der bereits erwähnte Richard Smith, der einen offenen Review Prozess und eine liberalere Publikationspolitik im Zeitalter des Internets befürwortet. Beim JMCR findet nach wie vor ein peer review vor einer Publikation statt. Die peers müssen sich aber zu erkennen geben, und ihre Kommentare werden mit dem Artikel zusammen auf der website publiziert. Dieser sog. open peer review process soll die Objektivität der Beurteilung fördern und die Zahl ungerechtfertigter Annahmen oder Ablehnungen von Manuskripten verringern. Wenn Sie eine Ablehnung erhalten, überlegen Sie sich mit Ihren Koautoren zusammen, ob Sie dem Herausgeber einen rebuttal schreiben wollen. Dies lohnt sich allerdings nur, wenn Sie gute Argumente haben. Wenn nicht, verzagen Sie nicht, sondern schreiben Ihren Artikel um, und senden inn an das nächste Journal. Bleiben Sie hartnäckig! Auch uns passiert das regelmässig. In einem Fall mussten wir sechsmal (!) eine Ablehnung akzeptieren. Aber schlussendlich wurde er doch publiziert, und vor allem wurde der Artikel von Mal zu Mal qualitativ besser, weil wir die guten Argumente oder berechtigten Kritikpunkte bei jeder neuen Version berücksichtigt haben.

\section{3) Öffentlicher Nutzen von Case Reports}

Der öffentliche Nutzen wurde bei den möglichen Themen bereits angesprochen und soll an dieser Stelle nur noch summarisch erwähnt werden.

Die wichtigste Rolle von Case Reports stellt die Erstbeschreibung von Krankheiten dar. Damit sind auch Erstbeschreibungen in neuem Kontext gemeint, oder die Publikation weiterer Fälle bei neuen Entitäten. Das heisst, dazu zählen auch Zweit-, Drittbeschreibungen usw.. In Unterstützung zu Public Health Massnahmen, Sentinella - Systemen und Postmarketing Analysen, wie auch der Pharmakovigilanz, Materiovigilanz, Hämovigilanz gehören auch Case Reports von unerwünschten Arzneimittelwirkungen, Zwischenfällen mit Geräten, Materialien und Blutprodukten zu den wichtigen und unverzichtbaren Domänen des Case Reports. Dies erhöht zweifelsohne die Sicherheit unseres Gesundheitswesens. Moderne Medien und das Internet mit open access ermöglichen auch den Aufbau einer weltweiten Database mit Case Reports allen nur denkbaren Inhalts, auch jenseits von Medline. Im Aufbau befindet sich zum Beispiel die Cases Database von BMC, unter: http://www.casesdatabase.com/.

\section{4) Persönlicher Nutzen von Case Reports}

Neben dem öffentlichen Nutzen gibt es auch einen persönlichen Nutzen für Sie selbst als Arzt und Mensch, weshalb Sie Case Reports schreiben sollten. Ein paar wichtige Punkte dazu sind in Abbildung 4 aufgelistet. 
a) Action research: Reflexion und Metakognition

Die Fachbegriffe Reflexion und Metakognition stammen aus der Psychologie und der Pädagogik, und sind uns Medizinern wenig geläufig. Ebenso sind die qualitative Sozialforschung und ihre Methoden bei uns nahezu unbekannt. Vom Selbstverständnis her sind die meisten von uns Naturwissenschaftler und agieren am liebsten mit Zahlen und sog. "hard facts“. Wer aber lange mit Menschen zu tun hat, und das einzelne Individuum in das Zentrum seines Tuns stellt, wie zum Beispiel jeder niedergelassene Hausarzt in der Praxis, oder jeder Arzt auf einer Visite im Spital, der weiss, wie viel sich nicht in Zahlen ausdrücken lässt. Die Beschreibung eines einzelnen Patienten in einem Case Report erlaubt jedem einzelnen von uns über seine eigene Aktivität nachzudenken. Was habe ich gemacht? Was haben wir richtig, was haben wir falsch gemacht? Was könnte ich beim nächsten Mal besser machen? Wie habe ich mit dem Patienten, mit meinen Kollegen kommuniziert? Wie kamen wir überhaupt auf unsere Diagnose? Und so weiter. Alle diese Fragen kann man in der Wissenschaftssprache der Aktionsforschung (action research) als reflektive und metakognitive Prozesse begreifen. Den Rahmen dieses CME würden wir sprengen, wenn wir hier noch tiefer schürfen würden. Deshalb lassen wir es mit diesen Andeutungen bewenden.

b) Individueller Patient: Empathie

Ein wichtiger Aspekt des Verfassens von Case Reports ist, dass sie diesem einen „Fall“, diesem Patienten, diesem individuellen Menschen Raum und Gewicht geben in Ihrem Wirken als Arzt. Mit anderen Worten zeigen Sie durch die Beschäftigung mit diesem einzelnen Patienten Empathie. Nur wenn Sie dem Patienten primär wegen der Einverständniserklärung erzählen, worum es Ihnen geht, zeigen Sie Interesse an ihm. In unserer Erfahrung sind die Patienten, die wir gefragt haben, fast immer sehr positiv eingestellt und fühlen sich geehrt, dass man über sie spricht, bzw., sie ernst nimmt.

c) Lebenslanges Lernen=Become a better doctor!

Durch das Aufschreiben des Fallberichtes, das Formulieren eines Textes werden Sie sich gründlicher und fokussierter mit diesem Patienten befassen. Sie lernen, auch durch das, was Sie vergessen haben und nachholen müssen für Ihren Fallbericht, wie Sie es beim nächsten Mal besser machen können. Kurz gesagt: Sie werden besser mit jedem einzelnen Case Report.

d) Teilnahme am wissenschaftlichen Diskurs

Etwas hochgestochen formuliert, aber inhaltlich doch korrekt, nehmen Sie durch Ihr Poster am Kongress, die Diskussionen mit den peers, die Korrekturen am Artikel und die Publikation aktiv Teil an der Medizin als Wissenschaft. Wenn auch noch so bescheiden, sind Sie Teil der Fortentwicklung des Fachgebietes. Sie nehmen Teil am wissenschaftlichen Diskurs. Und das kann für Sie sehr befriedigend sein: Sie haben einen Impact.

e) Narrative and listening competence / Healing

Ein letzter, etwas esoterisch klingender Punkt ist noch im Sinne der „narrative medicine“, dass sie durch Reflexion und Metakognition und das Aufschreiben bewusster werden, wie Sie kommunizieren, mit Ihren Patienten, mit Ihren Kollegen. Wie Allan Peterkin ausführt, haben Sie die Chance, durch das Schreiben Ihres Case Reports Ihre kommunikativen Fähigkeiten zu verbessern. Und diejenigen unter uns, die an die positive Kraft von Worten und guter Kommunikation glauben, können auch hoffen, dass bessere Ärztinnen und Ärzte auch bessere Heiler sein können. 


\section{Coda:}

Wir hoffen, Ihnen unsere Begeisterung für das Verfassen von Case Reports vermittelt zu haben mitsamt ein paar praktischen Tipps, damit auch Sie in nächster Zeit sagen können: „I published a Case Report!

Zum Abschluss lassen wir noch einmal Richard Smith zu Wort kommen, um insbesondere Hausärzte (GPs = general practitioners) dazu zu bewegen, von ihren Patienten und ihrer Arbeit zu berichten:

„So I urge you to write case reports, partly for the benefit of the world but perhaps even more for your own benefit. Once you've got started you'll come to love it".

Richard Smith 


\section{Beispiel eines Case Reports}

Q

Auswahl eines geeigneten Patienten

Im Frühjahr 2007 betreute einer der Autoren (M.S.) als Kaderarzt eine Patientin mit einer ungewöhnlichen Geschichte eines troponinpositiven Thoraxschmerzes mit blandem Koronarbefund in der Koronarangiographie. Die Patientin war damals 68 Jahre alt und nahm regelmässig Naratriptan ein bei einer seit Jahrzehnten bekannten Migräne.
Weg zum Case Report

Diesem Fall begegnete ich zum ersten Mal beim Rapport als Neuzugang über die Notfallstation.

Die Geschichte erschien mir bemerkenswert, so dass ich sie in mein Notizbuch eintrug.

Daraufhin gelang es mir, die zuständige Assistenzärztin für den Fall zu begeistern, und besprach mit ihr, diesen bei der nächsten SGIM - Jahresversammlung als Poster zu präsentieren.

Bei Gelingen der Präsentation stellte ich schon zu Beginn in Aussicht, den Fall mit inr zusammen als Case Report zu publizieren.

d

iteraturstudium und Arbeit

Die Assistenzärztin war/ist aussergewöhnlich interessiert und motiviert.

Die Literatursuche ergab einige Fälle von akuten Koronarsyndromen nach der Einnahme diverser Triptane bei Migränepatienten. Unser Fall war der erste mit Naratriptan.

\section{Abstract, Poster, Diskussionspunkt}

Der sogenannte „Test Drive“ des Falles als Poster bei der Jahresversammlung der SGIM war sehr erfolgreich, siehe auch: Weder C, Schneemann M. Triptane \& Troponin. $76^{\text {th }}$ Annual Meeting, May 2008, Lausanne.Schweizerisches Medizin-Forum 2008; 8:(Suppl. 40), 42 S (P67).

Mit dem Naranjo Algorithmus (5) erreichten wir einen Score von 6 Punkten was bedeutet, dass die Reaktion (Akutes Koronarsyndrom) eine wahrscheinliche Folge des eingenommenen Medikamentes war (Naratriptan). ihrer Migräne, und nimmt vorsorglich das gefässdilatierende Nifedipin zur Therapie ihrer arteriellen Hypertonie.

\section{直}

Die Suche nach einem geeigneten Journal richtete sich nach der Höhe des Impact Factors und der Bereitschaft, Case Reports zu publizieren. Dabei stiessen wir auf das Orphanet Journal of Rare Diseases (IF >3). Das Problem des „race for impact factors“ haben wir im Theorieteil das CME auch diskutiert. Leider akzeptiert dieses Journal seit 2010 keine Case Reports mehr.

Die Veröffentlichung gelang relativ reibungslos, die zwei Reviewers und der Editor verlangten lediglich „minor revisions“ und so gelang die Publikation zwei Jahre später, also im 2009, siehe Referenz 12. Die Publikationskosten von damals ca. 1500 SFR wurden durch unsere Institutionen, das UniversitätsSpital Zürich und die Universität Zürich (als institutional member der BMC Journals), übernommen. 


\section{${ }^{*}$ Fragen zu XY}

\section{*Frage 1}

Von wem stammt dieses Zitat: "always note and record the unusual. . .Publish it.

Place it on permanent record as a short, concise note.

Such communications are always of value"?
a) Rita Charon
b) William Osler
c) Marcel Proust
d) Fritz Reuter
e) Richard Smith

\section{*Frage 2}

Welches Akronym soll Ihnen beim Schreiben Ihres Case Reports helfen?
a) KISS
b) OSCE
c) TIMI
d) $T L ; D R$
e) YOLO

\section{*Frage 3}

Welche Schweizerische medizinische Fachzeitschrift akzeptiert explizit keine Case Reports?
a) Cardiovascular Medicine
b) PRAXIS
c) Primary Care
d) Schweizerisches Medizin Forum
e) Swiss Medical Weekly

\section{*Frage 4}

Nach welchen Kriterien oder mit welchem Score oder Algorithmus lassen / lässt sich die Wahrscheinlichkeit einer unerwünschten Arzneimittelwirkung abschätzen?
a) Curaçao Kriterien
b) Duke Kriterien
c) Fine Score
d) Geneva Score
e) Naranjo Algorithmus

\section{*Frage 5}

Wieviele „Case Reports“ wurden in den letzten zehn Jahren veröffentlicht und im PubMed aufgeführt?
a) 50 ‘000
b) $150 \times 000$
c) $250 ، 000$
d) $500 \times 000$
e) 1'000‘000 


\author{
${ }^{*}$ Autoren \\ PD Dr. med. Markus Schneemann, Klinik und Poliklinik für Innere Medizin, UniversitätsSpital \\ Zürich, Rämistrasse 100, 8091 Zürich \\ Dr. med. Fabio Ruggieri, Permanence Hauptbahnhof, Bahnhofplatz 15, 8021 Zürich
}

\title{
*Korrespondenzadresse
}

Markus Schneemann, Klinik und Poliklinik für Innere Medizin, UniversitätsSpital Zürich, Rämistrasse 100, 8091 Zürich; Email: markus.schneemann@usz.ch

\section{*Bibliographie}

1) Dieser CME-Beitrag basiert auf einem Workshop, den die Autoren an der 77. Jahresversammlung der Schweizerischen Gesellschaft für Innere Medizin am 13. Mai 2009 in Basel abgehalten haben. Als möglichen Conflict of Interest deklariert der Erstautor seine Funktion als Associate Editor beim Journal of Medical Case Reports und der PRAXIS, beide Autoren die Publikation von einigen Dutzenden von Case reports über die letzten fünfzehn Jahre in verschiedenen Sprachen, Formen und Zeitschriften.

2) Steurer J: Der Fallbericht - nicht nur Skurriles aus dem Raritätenkabinett. Praxis 2012; 101:215-6.

3) Smith R: Why GPs should write case reports. British Journal of General Practice 2009; 383.

4) Stäubli M, Suter J: Die Komplikationenliste der Schweizerischen

Gesellschaft für Innere Medizin. Schweizerische Ärztezeitung 2004;85:1109-16.

5) Lanctôt KL, Naranjo CA. Comparison of the Bayesian approach and a simple algorithm for assessment of adverse drug events. Clin Pharmacol Ther. 1995;58:692-8.

6) Osler W: The Quotable Osler. Philadelphia: American College of Physicians; 2003.

7) Kidd M, Hubbard C. Introducing journal of medical case reports. J Med Case Rep. 2007;1:1.

8) Kidd MR, Saltman DC. Case reports at the vanguard of 21 st century medicine. J Med Case Rep. 2012;6:156.

9) Peterkin A. Why we write (and how we can do it better). CMAJ 2010; 182:1650-2.

10) Charon R. Narrative and Medicine. N Engl J Med 2004;350:862-4.

11) Proust M. À la recherche du temps perdu : Guermantes, deuxième partie, p.148, Gallimard 1919 (fr.wikisource.org). In deutscher Übersetzung: „An die Medizin zu glauben wäre also der größte Wahnwitz, wofern es nicht ein noch größerer wäre, nicht an sie zu glauben, denn aus dieser Häufung von Irrtümern sind auf lange Sicht ein paar Wahrheiten hervorgegangen." (Guermantes, S. 418, Suhrkamp 2004, dt.: Eva Rechel-Mertens).

12) Weder CR, Schneemann M. Triptans and troponin: a case report. Orphanet J Rare Dis. 2009;4:15. 


\section{Antworten zu den Fragen zu XY aus PRAXIS Nr.}

\section{Frage 1}

Richtig ist die Antwort b).

William Osler gilt als einer der Väter der Inneren Medizin im angelsächsischen Raum. Er lebte von 1849 - 1919, stammte ursprünglich aus Ontario in Kanada, lehrte in Philadelphia und Baltimore in den Vereinigten Staaten von Amerika und war zuletzt Regius Professor of Medicine in Oxford, Vereinigtes Königreich.

Ad a, c, d, e). Von diesen Personen stammen die anderen Zitate in diesem CME-Artikel. Rita Charon, geboren 1949, ist Allgemeininternistin und leitet an der Columbia University in New York das Program in Narrative Medicine.

Marcel Proust (1871 - 1922) ist einer der führenden Schriftsteller des 20. Jahrhunderts. Sein Hauptwerk ist der mehrbändige Roman "À la recherche du temps perdu“.

Fritz Reuter (1810 - 1874) ist ein bedeutender Dichter der niederdeutschen Sprache. Das im Eingang zum CME angeführte Zitat stammt aus seinem Buch Ut mine Stromtid (Aus meiner Volontärszeit) von 1862.

Richard Smith, geboren 1952, war lange Jahre (1991 - 2004) Herausgeber des British Medical Journal. Er ist ein scharfer Kritiker der Publikationsindustrie in der Medizin und ein Befürworter der Publikation von Case Reports.

\section{Frage 2}

\section{Richtig ist Antwort a).}

Das gesuchte Akronym („sprechende Abkürzung“) ist KISS, welches steht für: „Keep it simple, stupid." Es soll das Schreiben von Texten erleichtern helfen.

Ad b, c, d, e). OSCE steht für "Objective structured clinical examination". Diese Art der Prüfung mit Schauspielerpatienten wird auch bei Eidgenössischen Medizinalprüfung eingesetzt. TIMI steht für Thrombolysis in Myocardial Infarction, eine berühmte Gruppe von Studien, initiiert 1984 von Eugene Braunwald und Mitarbeitern in Boston. Viele medizinische Studien arbeiten mit Akronymen. TL;DR steht für: " too long; didn't read”. Insbesondere im Zeitalter von Email, Facebook und Twitter sind Akronyme auch bei Jugendlichen (die "Generation y“) sehr beliebt. YOLO steht für: „You only live once“ und wurde 2012 in Deutschland zum Jugendwort des Jahres gewählt.

\section{Frage 3}

\section{Richtig ist Antwort e).}

Das Swiss Medical Weekly wünscht gemäss ihren Instructions for authors explizit keine Case Reports.

Ad a, b, c und d). Diese aus der Schweiz stammenden Zeitschriften akzeptieren Case Reports. Unsere Zeitschrift, PRAXIS, bevorzugt „praxisrelevante Fälle, keine Raritäten“. Das Schweizerische Medizin-Forum akzeptiert Fallberichte als „Coup d'œil, Leserbrief oder bei besonderem Fortbildungswert - unter der Rubrik Der besondere Fall“. Cardiovascular Medicine publiziert Images, EKGs und "unusual or surprising and significant observations". Primary Care publiziert "Kolumnen, «Billets d'humeur» oder narrative Berichte."

\section{Frage 4}

\section{Richtig ist Antwort e).}

Die Wahrscheinlichkeit einer unerwünschten Arzneimittelwirkung kann mit einem von Claudio Naranjo, einem Pharmakologen aus Toronto 1981 zum ersten Mal formulierten und publizierten Algorithmus beschrieben werden:

http://farmacologiaclinica.info/scales/Naranjo_scale

Ad a, b, c, d). Die Curaçao-Kriterien sind klinische Kriterien zur Diagnose der hereditären hämorrhagischen Teleangiektasien (HHT), dem Morbus Osler-Weber-Rendu. Die Duke Kriterien helfen bei der Diagnose einer infektiösen Endokarditis. Der Fine Score ist ein prognostischer Index zur Abschätzung von Morbidität und Mortalität bei ambulant erworbenen Pneumonien. Der Geneva Score ist ein dem Wells Score verwandtes Instrument zur Diagnose einer Lungenembolie. 


\section{Frage 5}

Richtig ist Antwort d).

Im PubMed finden Sie mit dem Suchwort „Case Report“ knapp über 500‘000 hits über die letzten zehn Jahre. Hinzuzählen müsste man noch die dort nicht aufgeführten Artikel, vor allem in nicht-englischer Sprache. 